\title{
JOCHEN FRANZKE
}

Poczdam

\section{Die Eurokrise und ihre strategischen Folgen für Europa}

Drastische Worte über Europas Zustand fand unlängst das Zentrum für Sicherheitsstudien der Eidgenössischen Technischen Hochschule Zürich in seiner bemerkenswerten Studie Strategic Trends 2012: „Die Schuldenkrise hat strategische Folgen für Europa. Die Rolle der EU als Stabilitätsanker ihrer Mitgliedstaaten wurde geschwächt. Intraeuropäische Machtverschiebungen, die politische Fragmentierung, eine Re-Nationalisierungsdynamik und das sinkende Vertrauen der Öffentlichkeit in die EU haben das europäische Projekt in die größte Krise seiner Geschichte geführt" ${ }^{\text {"1 }}$. Selbst wenn man diese weitreichende Einschätzung nicht teil, kommt man nicht umhin festzustellen, dass die seit dem Frühjahr 2010 anhaltende, nicht bewältigte Staatsschuldenkrise im Euroraum die Europäische Union nachhaltig verändert hat. Im Folgenden soll der Versuch unternommen werden, die`se Veränderungen und deren Auswirkungen auf die Rolle Europas in der Welt zu skizzieren.

\section{Wie alles begann}

Die Staatsschuldenkrise nahm ihren Ausgangspunkt, als einige Mitgliedstaaten der Eurozone den Zahlungsverpflichtungen aus ihrer Verschuldung ohne Unterstützung Dritter nicht mehr nachkommen konnten. Am 23. April 2010 beantragte Griechenland, um einen drohenden Staatsbankrott zu verhindern, als erstes Land offiziell EU-Hilfe. Wenig später konnten Irland und Portugal ihre Staatsschulden ebenfalls nicht mehr bedienen. Auch Italien und Spanien hatten - wenn auch anfangs in geringerem Maße - Probleme, am Kapitalmarkt Kredite aufzunehmen. Alle diese Länder zählen daher bis heute zu den Krisenstaaten.

Die tieferliegenden Krisenursachen sind vielfältig: Zuallererst können diese im globalen kapitalistisch geprägten Wirtschaftssystem und seinen unvermeidbaren ,finanziellen Instabilitäten" verortet werden. Dazu gehört vor allem, dass die Banken (zumindest die sogenannten systemrelevanten) sich de facto jeglicher politischer Kontrolle entzogen haben. Katalysator der Euro-Krise ist die bis heute ebenfalls nicht bewältigte globale Finanzkrise, die im Frühjahr 2007 als Immobilienkrise in den USA begann und mit dem Zusammenbruch der amerikanischen Großbank Lehman Brothers im September 2008 ihren vorläufigen Höhepunkt hatte. Hinzu kommen spezifische Ursachen in den von der Staatsschuldenkrise betroffenen EU-Mitgliedsländern, die aus unterschiedlichen inneren Fehlentwicklungen herrühren.

${ }^{1}$ Center for Security Studies Center for Security Studies (ed.), Strategic trends 2012. Key Developments in Global Affairs, Zürich 2012, s. 55. 
Immer sichtbarer wird allerdings, dass die europäische Staatsschuldenkrise durch Geburtsfehler bei der Schaffung der Wirtschafts- und Währungsunion in den 1990er Jahren mit verursacht wurde. Der im Dezember 2009 in Kraft getretenen Lissabon-Vertrag konnte diese Defizite nicht beseitigen. Im Gegenteil, dieser hat die hohen in ihn gesetzten Erwartungen der Verbesserung der Handlungsfähigkeit der Union nicht erfüllt.

\section{Eurokrise verstärkt Europas Fragmentierung}

Nachdem sich die EU dafür entschieden hatte, die Krisenstaaten in der Eurozone zu halten und auf den klassischen Weg von Staatsbankrotten zu verzichten, griff die Staatsschuldenkrise auf die europäische Währungszone über. Seither kann auch von der „Euro-Krise“ gesprochen werden, die in der Folge sowohl die Eurozone als auch das europäische Integrationsprojekt erschütterte. Der eigentliche Patient in der Eurokrise sind daher nicht mehr in erster Linie die oben genannten Krisenstaaten, sondern die Europäische Union selbst.

Die beklagte politische Fragmentierung der EU hat viele Fassetten. Ich möchte auf zwei eingehen: Erstens hat die Euro-Krise die Kluft zwischen den strategischen Vorstellungen über die Zukunft der Union zwischen den EU-Mitgliedsstaaten deutlich vertieft. Einige erwarten von einem Integrationsschub durch Souveränitätsübertragungen auf Europa in der Finanz- und Haushaltspolitik den Ausweg aus der Krise. Andere wollen gerade die Haushaltspolitik als nationalstaatliches Steuerungsinstrument behalten und sehen in der Re-Nationalisierung politischer Entscheidungen den richtigen Weg aus der Krise. Die Zahl jener EU-Mitgliedsstaaten ist deutlich gewachsen, „die weitere Integrationsschritte und Kompetenzübertragungen an die EU kategorisch ablehnen oder gar die Re-Nationalisierung einzelner Bereiche fordern“" ${ }^{2}$.

Zweitens trägt die durch die Euro-Krise erzwungene weitere Institutionalisierung der Euro-Zone zur politischen Fragmentierung in der EU bei. Die Funktionsweise der seit 1998 bestehenden Euro-Gruppe als EU-Gremium ist seit dem Lissabon-Vertrag geregelt ${ }^{3}$. Diese Gruppe, der zurzeit 17 der 27 EU-Länder angehören, soll deren Steuerund Wirtschaftspolitik koordinieren sowie den Euro-Stabilitätspakt überwachen, ohne allerdings Entscheidungsbefugnisse zu besitzen. Im Zuge der Staatsschuldenkrise beginnt sich die Euro-Zone seit Ende 2011 institutionell zu verselbständigen (z.B. durch eigene Gipfeltreffen und die informelle Frankfurter Runde). Diese Entwicklung hebelt das institutionelle Gefüge des Lissaboner Vertrages de facto weiter aus ${ }^{4}$. Dies könnte zu einer dauerhaften Sollbruchstelle in der EU führen, da die angestrebte Eu-

2 L. Brozus, D. Kietz, N. von Ondarza, Die Entwicklung des EU-Systems zwischen Reformdruck und Integrationsmüdigkeit Möglichkeiten und Grenzen des Pragmatismus, in: A. Bendiek, B. Lippert, D. Schwarzer, Entwicklungsperspektiven der EU. Herausforderungen für die deutsche Europapolitik, „SWP-Studien“, Berlin 2011, s. 9.

3 Siehe Art. 137 des Vertrages über die Arbeitsweise der Europäischen Union mit Verweis auf dessen Protokoll Nr. 14 (siehe http://www.aeuv.de/).

4 Bereits im Mai 2010 hatte der Europäische Rat auf seiner Sondersitzung zur Staatsschuldenkrise die No-Bailout-Klausel des Lissabon-Vertrages de facto außer Kraft gesetzt. 
ro-Mitgliedschaft aller EU-Mitgliedsstaaten durch die Euro-Krise in weite Ferne gerückt ist. Ob die geplante Re-Integration der neuen Institutionen der Eurogruppe in die EU gelingen kann, steht in den Sternen. Vor allem aber hat das gegenseitige Vertrauen der Akteure - die wichtigste Währung in der Europäischen Union - erheblich gelitten.

\section{Trilemma der europäischen Integration}

Unter diesen Umständen verschärfen sich die Zielkonflikte in der Union. Mit dem Begriff des „Trilemma(s) der europäischen Integration“, das die Zieldimenionen Erweiterung, Vertiefung sowie Demokratie betrifft, haben die Politikwissenschaftler Höppner, Schäfer und Zimmermann das Problem auf den Punkt gebracht. Sie kommen zu dem ernüchternden Ergebnis: „Von den drei Zielen der EU lassen sich stets nur zwei gleichzeitig erreichen, auf Kosten des jeweils dritten Ziels ${ }^{\text {‘5 }}$.

Dieser Befund ist diskussionswürdig. Hier ein kurzer Blick auf alle drei Zieldimensionen. Beginnen wir mit der Erweiterung. Deren Ziel war es, durch Ausweitung der EU politische, rechtliche und ökonomische Stabilität zu exportieren ${ }^{6}$. In Bezug auf die Staaten Mittelosteuropas ist dies durchaus gelungen. Die ungebremste Erweiterungsdynamik der EU hat schließlich zu einem dysfunktional großen und heterogenen Wirtschafts- und Währungsraum geführt.

Das Thema der EU-Erweiterung ist keineswegs vom Tisch. Kroatiens wird Mitte 2013 der 28. Mitgliedsstaat der EU; Islands Beitritt ist wahrscheinlich. Die zumeist erweiterungsmüden europäischen Eliten sind allerdings über künftige Erweiterungen der EU uneinig. Besonders problematisch ist dies für den Westbalkan, wo die EU kein über die Bewahrung des Status quo hinausgehendes Konzept der weiteren Stabilisierung und Demokratisierung besitzt ${ }^{7}$. Dabei wollte Europa gerade in dieser latenten Konfliktzone nachweisen, dass es über die seit den blutigen Balkankriegen der 1990er Jahren geforderte Fähigkeit verfügt, seine inneren Konflikte selbst zu lösen. Ein Scheitern der EU in diesem Raum könnte dort zu einer gefährlichen dauerhaften Instabilität führen. Für die künftige globale Rolle der EU noch dramatischer ist allerdings die fehlende Strategie gegenüber einer dauerhaften Anbindung der Türkei und der Ukraine an Europa.

\section{Staatsschuldenkrise und Vertiefung}

Die zweite Zieldimension des Trilemmas der europäischen Integration betrifft die Vertiefung der europäischen Integration. Funktionelle Sachzwänge prägen die EU von Anfang an und haben die Integration schrittweise auf weitere Politikfelder ausgreifen lassen und damit vorangebracht. Im Laufe der Zeit sind aber statt dieser Sachzwänge

5 „Frankfurter Allgemeine Zeitung“, 17.4.2012.

6 Bereits die Erweiterungsrunde 2007 um Rumänien und Bulgarien zeigte jedoch das Scheitern dieses Ansatzes, jedenfalls in den zugrundegelegten Zeiträumen und mit den eingesetzten Instrumenten.

7 Siehe: A. Despot, D. Reljić, Dušan, G. Seufert, Zehn Jahre Einsamkeit. Zur Überbrückung der Pause im Erweiterungsprozess der Europäischen Union sollten dem Westbalkan und der Türkei praktische Integrationsschritte angeboten werden, „SWP-Aktuell“, A 23, April 2012. 
- darauf weisen Höppner, Schäfer und Zimmermann zurecht in - die Präferenzen der politischen, rechtlichen und wirtschaftlichen Eliten Europas sowie die Eigeninteressen der europäischen Bürokratie zum Tragen gekommen. Heute muss mithin genauer hingeschaut werden, was echte Sachzwänge sind und was nicht ${ }^{8}$.

Dies gilt auch und gerade für die Eurokrise. Diese hat die makroökonomischen Ungleichgewichte in der EU verstärkt, in der unterschiedliche volkswirtschaftliche Strukturen, differenzierte Verschuldungskulturen und unterschiedliche Systeme der Lohnfindung aufeinandertreffen. Klassische Lösungen wie eine nach Ländern differenzierte Geldpolitik sowie die Möglichkeit der Wechselkursanpassung sind in einer Wahrungsunion jedoch nicht mehr gegeben. Der Politik bleibt daher nur die Alternative, diese aufrechterhalten oder aufzulösen. Die europäischen Eliten haben sich offenbar für ersteres entschieden.

Die dritte Zieldimension des Trilemmas der europäischen Integration betrifft die für westliche Länder zentrale Frage der Demokratie. Höppner, Schäfer und Zimmermann kommen zu dem Ergebnis, dass die Demokratie „der Verlierer der Erweiterungs- und Vertiefungspolitik der vergangenen beiden Jahrzehnte (ist)“. Die Eurokrise hat dies weiter verschärft. Weiter schreiben sie zutreffend: „(E)ine heterogene Union, die auf allen erdenklichen Politikfeldern Kompetenzen beansprucht, höhlt die Demokratie der Mitgliedstaaten schleichend aus, ohne ihrerseits demokratisierbar zu sein“"9.

Die modernen Gesellschaften Europas verlangen nach neuen, über die technokratische Legitimationsrhetorik hinaus gehende Begründungen für die Relevanz der EU. Annegret Bendiek bringt es auf den Punkt: „Die alte Gleichsetzung von ,mehr' Europa mit einem demokratischeren und leistungsfähigeren Europa ist historisch überholt ${ }^{\star 10}$. Die Ausdehnung der Kompetenzen nationaler Parlamente und die Ausweitung des Mitentscheidungsverfahrens durch den Lissabon-Vertrag seien zwar Schritte in die richtige Richtung. Für sich genommen liefern sie aber „noch keine abschließende Antwort auf die Frage nach dem Ort und der Gestalt der Demokratie in Europa“"11. Ohne klare Antwort auf diese Frage wirkt der Ruf nach ,mehr Europa“ eher bedrohlich als beruhigend.

\section{Eurokrise und Deutschlands europäische Rolle}

Die Rolle Deutschlands in der EU ist durch die Staatsschuldenkrise deutlich aufgewertet worden. Es ist zum unbestrittenen EU-Leader aufgestiegen. Eine Schlüsselrolle wird von ihm vor allem bei der konstitutionellen Weiterentwicklung der Union und der Durchsetzung neuer Entwicklungsoptionen erwartet ${ }^{12}$. Auf damit verbundene Nebenwirkungen weist die schon mehrfach zitierte Schweizer Studie hin: „Deutschland hat diese Führungsrolle nicht gesucht. Dennoch führte die Euro-Krise zu eine Rückkehr der fast vergessenen , deutschen Frage', wenn auch in einer wirtschaftlichen Variation.

\footnotetext{
8 Siehe: „Frankfurter Allgemeine Zeitung“, 17.4.2012.

Ibidem.

10 L. Brozus, D. Kietz, N. von Ondarza, op. cit., s. 8.

11 Ibidem, s. $6 \mathrm{f}$.

12 Siehe: L. Brozus, D. Kietz, N. von Ondarza, op. cit., s. 8.
} 
Europas gemeinsame Währung sollte das jahrhundertealte Problem lösen, dass Deutschland für ein europäisches Kräftegleichgewicht zu stark, zugleich für eine Vorherrschaft zu schwach ist. Es zeigt sich nun, dass die Währungsunion diese Frage tatsächlich wiederbelebt hat “'13.

Dies stellt vor allem die deutsche Politik vor ein ernstes politisches Problem. Mehr Führungsstärke Deutschlands wird in Europa vielfach gefordert, wie z.B. durch den polnischen Außenminister Sikorski im November 2011 in Berlin. Die Dominanz Deutschlands wird zugleich nach wie vor in vielen EU-Ländern kritisch gesehen bzw. gefürchtet. Um daraus keine neue Gefahr für die Einheit Europas werden zu lassen sind zwei Dinge notwendig: eine Kultur der Zurückhaltung bei den deutschen Eliten und die Existenz von politischen Gegengewichten in der EU.

\section{Deutschland sucht ein Europakonzept}

Der innenpolitische Umgang mit der Staatsschuldenkrise in Deutschland ist widersprüchlich. Formell unterstützt eine staatstragende Superkoalition bestehend aus den Regierungsparteien und den Oppositionsparteien SPD bzw. Grünen die angeblich alternativlose ad hoc Eurorettungspolitik der Regierung Merkel. In allen Abstimmungen im Bundestag zur Eurokrise hatte diese bislang stets eine stabile zwei-Drittel-Mehrheit ${ }^{14}$. Nur die Linkspartei hat sich in allen Abstimmungen dieser Position verweigert.

Schaut man jedoch genauer hin, ist von einem europapolitischen Konsens auf der konzeptionellen Ebene in Berlin wenig zu spüren. Die Ursache für den im restlichen Europa verbreiteten Vorwurf, Berlin führe in der EU zu wenig, liegt ja gerade im Fehlen eines echten europapolitischen Zukunftsprogramms mit klaren Zielvorgaben und konkreten Umsetzungsdetails. Im Gegenteil, die Auseinandersetzung über den weiteren Weg der europäischen Integration haben sich innerhalb der politischen Elite Deutschlands - teilweise quer zu den Parteilinien - seit Ausbruch der Eurokrise dramatisch verschärft.

Drei Ansätze stoßen dabei aufeinander: Erstens hat die Euro-Krise zu einer Mobilisierung der Verfechter der Vereinigten Staaten von Europa in Deutschland geführt. Typisch dafür ist z.B. folgende Einschätzung des Programmdirektors Europa der Bertelsmann-Stiftung Fritz-Vannahme: „Mit dem bestehenden Gemeinwesen wird mehr nicht zu erreichen sein. Die Europäische Union steht auf der Schwelle zu den Vereinigten Staaten von Europa ${ }^{\text {“15. }}$.

13 Center for Security Studies 2012, op. cit., s. 63.

14 Typisch ist das Abstimmungsverhalten zum Fiskalpakt am 29.6.2012. Zugestimmt haben dem entsprechenden Gesetz insgesamt 78\% der Abgeordneten. Dagegen stimmten 1\% der CDU-Abgeordneten, 2\% der CSU-Abgeordneten, 4\% in der FDP-Fraktion, 16\% in der SPD-Fraktion, 13\% in der Fraktion der Grünen/Bündnis 90 und die gesamte Fraktion der Linkspartei (nach Angaben von abgeordnetenwatch.de).

15 J. Fritz-Vannahme, Für die Vereinigten Staaten von Europa, ,Spotlight Europe“ 2011, Nr. 3, s. 1. Mehrere Minister der Bundesregierung haben sich öffentlich für diesen Ansatz ausgesprochen, darunter Außenminister Westerwelle, der erklärte: „Ich will die Vereinigten Staaten von Europa noch erleben“. („Focus“, 21.8.2011). 
Zweitens ist jene Gruppe aktiver geworden, die weitere Souveränitätsübertragungen auf die EU stoppen will. Die EU - so ihr Credo - darf kein Staat werden. Dabei knüpfen sie vor allem an das Urteil des Bundesverfassungsgerichts zum Lissabon-Vertrag vom 30.6.2009 an. Typisch dafür ist z.B. die Stellungnahme des Staatsrechtlers und ehemaligen Verfassungsrichters Paul Kirchhof, der feststellte: „Deutschland bleibt ein souveräner Staat; es behält auch die Verantwortlichkeit für seine Wirtschaftsstruktur und sein Wirtschaftsrecht. Die Vereinigten Staaten von Europa wird es unter Geltung des Grundgesetzes nicht geben“"16.

Schließlich finden sich drittens die Verfechter einer weiteren Vertiefung der Integration durch Schaffung einer politischen Union als Antwort auf die Eurokrise. Bundeskanzlerin Merkel, die „mehr Europa“ wagen will, gehört offenbar dazu. Sie hat es bislang allerdings hervorragend verstanden, die Öffentlichkeit über Einzelheiten ihres Konzepts im Dunkeln zu lassen. Sie betrachtet dies offenbar als Langzeitprojekt mit der Fiskalunion als ersten Schritt. Klarere Vorstellungen über die mögliche Struktur einer politischen Union finden sich bei hingegen bei Bundesfinanzminister Schäuble, der dafür eintritt ,jetzt eine politische Union Europas schaffen, weil wir es in den 90iger Jahren nicht konnten... Aber wir wollen nicht einen europäischen Superstaat ${ }^{\star 17}$. Allerdings ist dieser Ansatz in seiner Allgemeinheit auch gut geeignet, als ideologische Verbrämung eines simplen weiteren „Durchwurstelns“ durch die Krise herzuhalten.

Sowohl die Anhänger der „Vereinigten Staaten von Europa“ als auch die Gegner eines europäischen Superstaates sind in Deutschland weiterhin politisch in der Minderheit. „Mehr Europa“ durch die Schaffung einer politischen Union könnte sich als neuer Konsens und Konsequenz aus der Euro-Krise durchaus durchsetzen. Dazu braucht es aber ein tragfähiges Konzept mit Antworten im Detail, die weit über die fiskalischen Fragen hinausgehen. Erst wenn dieses auf dem Tisch liegt, kann eingeschätzt werden, ob „mehr Europa“ wirklich die Lösung der Euro-Krise darstellt.

\section{Europas Abstieg beschleunigt?}

Wie immer in Zeiten der Krise stellt sich die grundsätzliche Frage der Sinnhaftigkeit der europäischen Integration und der Rolle des alten Kontinents in der Welt von morgen. Die schon erwähnte Studie „Strategic Trends 2012“ kommt z.B. zu dem erschreckenden Schluss, dass durch diese Krise die Rolle der EU als Stabilitätsanker in ganz Europa beschädigt sei und sich dessen langfristiger globaler Abstieg beschleunigt habe $^{18}$. Ähnlich fällt übrigens auch die Antwort des amerikanischen Historikers Walter Laqueur aus, der bereits 2009 die finanzielle und politische Krise Europas prophezeit hatte $^{19}$. Laqueur sieht auch aktuell keine besseren Aussichten für den ,troubled conti-

16 „Frankfurter Allgemeine Zeitung“, 30.6.2009.

17 Schäuble 2012 in seiner Rede anlässlich der Verleihung des Karlspreises am 17. Mai 2012. Siehe: Rede anlässlich der Verleihung des Internationalen Karlspreises zu Aachen am 17. Mai (http://www.wolfgang-schaeuble.de/index.php?id=30\&textid=1524\&page=1).

${ }_{10}$ Center for Security Studies 2012, op. cit., s. 55.

19 Siehe: W. Laqueur, After the Fall: The End of the European Dream and the Decline of a Continent, New York 2009. 
nent“, der „nicht mehr die Kraft und den politischen Willen zu haben (scheint), eine wirklich wichtige Rolle auf der Weltbühne zu spielen “20.

Nun ist globale Politik kein Nullsummenspiel. Europas wahrscheinlicher Abstieg ist zuallererst ein relativer: sein Einfluss nimmt wegen des Aufstiegs anderer Mächte ab. Ob die Staatsschuldenkrise diesen Abstieg beschleunigen wird ist offen. Europa steckt immer noch mitten in der Krise, alle Analysen dazu - auch diese - sind nur Momentaufnahmen. Die zitierten Katastrophenszenarios der Eidgenossen oder von Walter Laqueur haben eine gewisse Plausibilität. Es besteht aber auch eine reale Chance, durch einen erfolgreichen Umbau der EU die Krise erfolgreich zu meistern. Nur ein konzeptionsloses „weiter so" scheint in die Katastrophe zu führen.

Nicht vergessen werden sollte dabei aber, dass die vielfältigen Probleme der Welt im 21. Jahrhundert - angefangen von der Proliferation von Massenvernichtungswaffen, dem Klimawandel, dem internationalen Terrorismus bis hin zu den labilen Finanzmärkten - die Möglichkeiten der Nationalstaaten übersteigen. Dies gilt auch für die - so die neue Berliner Wortwahl - „neuen Gestaltungsmächte“ wie China, Indien, Brasilien, Mexiko und Südafrika. Sicher ist der Aufstieg dieser Mächte unübersehbar und wird sich fortsetzen, wobei auch diese gewaltige innere sozio-ökonomische Krisen zu bewältigen haben. Bei der notwendigen Strukturbindung über die Nationalstaaten hinaus, der Vernetzung und dem dazu nötigen Multilateralismus ist Europa diesen Mächten weiterhin voraus.

Der bisherige Umgang mit der europäischen Schuldenkrise hat zweifellos das Vertrauen in die überlegene Problemlösungsfähigkeit demokratischen Regierens des Westens erschüttert. Hier liegt das eigentliche Problem. Es sollte einiges passieren, um dieses Vertrauen wiederherzustellen. Die konfliktbeladene Umbruchszeit in Europa ist noch nicht zu Ende.

\section{Summary}

\section{The euro crisis and its strategic implications for Europe}

The author claims that the eurozone crisis has generated the most difficult situation European integration has faced since the 1950s. The crisis will hinder the enlargement of the European Union with new members and the process of EU democratization. The role of Germany in the EU has been excessively increased, and Berlin will take advantage of this, persuading other partners to adopt German solutions in terms of recovery from the crisis. With the exception of the left-wing party (Die Linke), the remaining German political parties support the government's point of view. The eurozone crisis has resulted in the global deterioration of the EU's image, contributing to a decline in its importance and impact.

20 „Welt online“, 27.6.2012. Sie auch Laqueur 2012. 
